http://jmscr.igmpublication.org/home/ ISSN (e)-2347-176x ISSN (p) 2455-0450

crossref DOI: https://dx.doi.org/10.18535/jmscr/v8i1.38

Journal Of Medical Science And Clinical Research

\title{
Study on Etiopathogenesis of Stridor in Neonates, Infants and Children in Tertiary Care Hospital
}

\author{
Authors \\ Dr Leela Sowmya Saladi ${ }^{*}$, Dr S. Surya Prakasa Rao², Dr K.V Madhavilatha ${ }^{3}$, \\ Dr T.V.S.S.N. Leelaprasad ${ }^{4}$ \\ ${ }^{1}$ Junior Resident of ENT, Department of ENT, Andhra Medical College, Visakhapatnam, India \\ ${ }^{2}$ Professor of ENT, Department of ENT, Andhra Medical College,Visakhapatnam, India \\ ${ }^{3}$ Senior consultant, Department of Gyanecology, Andhra Medical College, Visakhapatnam, India \\ ${ }^{4}$ Assistant Professor, Department of ENT, Andhra Medical College,Visakhapatnam, India \\ *Corresponding Author \\ Dr Leela Sowmya Saladi
}

\begin{abstract}
Background: A child with stridor typically induces significant anxiety in the carer and should be a clinical priority for the medical professional. In the acute situation the paediatric airway can deteriorate rapidly and this can be a real challenge for the paediatrician, otorhinolaryngologist and anaesthesiologist. Stridor which is the main symptom of upper airway obstruction in infants, can be congenital or acquired, acute or chronic and the Pathologies can be located from the nose down to the trachea. Common causes include laryngomalacia, vocal cord palsy, subglottic stenosis, tracheal anomaly, laryngeal cleft, vascular and lymphatic malformation, laryngeal papillomas, craniofacial abnormalities and even head and neck tumours. In this study various etiological causes of stridor in neonates, infants and children are evaluated. It is important to evaluate the cause of stridor as the type and cause of the anatomical and functional abnormality determines the prognosis.

Materials and Methods: Cases (neonates,infants and children) presented with stridor to ENT OPD,to ENT casualty and referral cases from pediatrics department of King George Hospital,Visakhapatnam were evaluated and investigated during the period of one year from March 2017 to March 2018. The cause of stridor is evaluated by careful history taking, thorough physical examination and imaging studies and endoscopy. Imaging studies include digital x-ray,plain CT, CECT, MRI. Endoscopic studies include direct examination of larynx and trachea by rigid as well as flexible endoscope.

Results: Out of 22 patients 14 patients presented with congenital causes and 8 patients presented with acquired causes of stridor. Most common cause (45\%,22 patients) of stridor in this study group is laryngomalacia. 3 cases had vocal cord palsy. 2 cases presented with foreign body in trachea and one with foreign body in glottis. 2 cases had post intubation injury, 1 case with subglottic web, 1 case with squamous papilloma, 1 case with subglottic haemangioma, 1 case was diagnosed as diphtheria. Immunisation history was normal in all patients except in one case which we encountered Diptheria.

Conclusion: Stridor is an important condition, in many cases implying an impending disaster with a very compromised airway. Evaluation should start from careful history taking and clinical examination. Persistent symptoms deserve referral to otorhinolaryngologists for flexible and rigid endoscopy.

Neonates with stridor should be managed in a multidisciplinary setting, by clinicians familiar with the intricate physiology of these children, and with access to the multitude of medical and surgical investigative and therapeutic options required to provide first-rate care.

Keywords: Neonate, Infant, Children, Stridor, Foreign Body, Laryngomalacia.
\end{abstract}




\section{Introduction}

Noise originating in the larynx or trachea is typically high-pitched and termed 'stridor'. It may have a musical quality. In contrast, the lowpitched snoring type of noise made by naso- and oropharyngeal obstruction (and rarely by the supraglottic larynx) has a rougher quality and is best described as 'stertor". The word stridor is derived from Latin word stridulus which means creaking,whistling or grating. Inspiratory stridor is due to obstruction at the supraglottic and glottis level of upper airway, biphasic stridor is due to obstruction at the subglottic level, and expiratory stridor is due to obstruction in the trachea ${ }^{6}$. Stridor in neonates can be due to congenital or acquired causes. Though the commonest cause of stridor in neonates is laryngomalacia, there are secondary airway lesions (SALs) and other causes such as vocal cord palsy, subglottic stenosis, tracheal anomaly, laryngeal cleft, vascular and lymphatic malformation, laryngeal papillomas, craniofacial abnormalities, and even head and neck tumours ${ }^{2,3}$. Persistent stridor deserves careful assessment and proper investigation instead of just labeling it as a benign condition such as laryngomalacia.Various radiological tests are available for evaluation of stridor.

Plain anteroposterior (AP) and lateral views of the neck, digitally enhanced to demonstrate the subglottis, oropharynx and nasopharynx, are rapid, inexpensive tests that do not require sedation or anaesthesia. Foreign bodies that do not cause complete lower airway obstruction, those that are radiolucent and those located in the larynx represent particular diagnostic challenges ${ }^{7}$. Airway fluoroscopy has been found to be highly specific in the diagnosis of both laryngotracheomalacia and fixed airway lesions, but its sensitivity is poor; it has not been proven to be a useful screening tool ${ }^{8}$. Airway fluoroscopy can be combined with a contrast swallow looking for vascular compression and aspiration. Contrast swallows are of limited diagnostic value in the investigation of paediatric stridor and may be best employed following endoscopy, particularly in cases in which a vascular ring or tracheooesophageal fistula is suspected ${ }^{9}$. Bronchography may be performed using non-ionic contrast media. It requires expertise and is associated with risk, particularly in infants and those prone to apnoea. It is particularly useful for the lower airway, demonstrating tracheobronchial stenosis and malacia ${ }^{10}$. Both computerized tomography (CT) and magnetic resonance imaging (MRI) can demonstrate the configuration of thoracic vasculature in cases of extrinsic tracheal compression and thus are particularly useful postendoscopy ${ }^{11}$. Investigations need to be carefully selected on the basis of the history and examination.

\section{Materials and Methods}

An Observational study on etiopathogenesis of stridor in neonates, infants and children was conducted durin the time period between March 2017 and March 2018 in a tertiary health care centre. Cases presented to ENT OPD and referral cases from paediatrics department were evaluated during this period. Children between 0-14 yrs of age were included in the study.Prior to evaluation of cause of stridor tracheostomy is done in required cases. The cause of stridor is evaluated by careful history taking, thorough physical examination and imaging studies and endoscopy.

\section{Result}

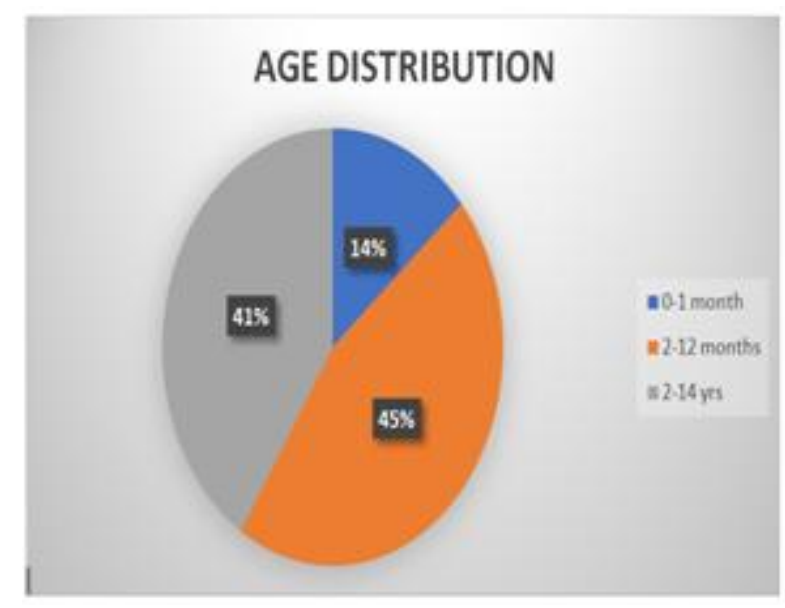


GENDER DISTRIBUTION

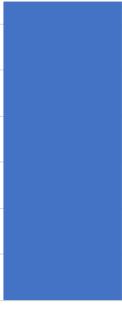

Male

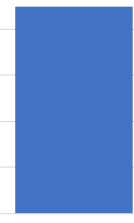

Female

Site of involvement

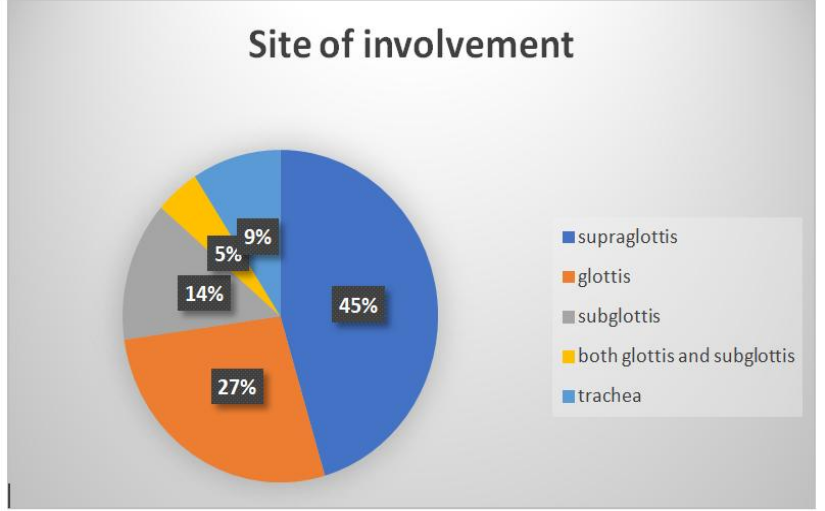

\section{Cause of stridor}

\begin{tabular}{|l|c|}
\hline Cause of stridor & No.of patients \\
\hline Congenital & 14 \\
\hline Acquired & 08 \\
\hline Total & 22 \\
\hline
\end{tabular}

Congenital causes of stridor

\begin{tabular}{|l|c|}
\hline Cause & No.of patients \\
\hline Laryngomalacia & 10 \\
\hline Unilateral vocal cord palsy & 02 \\
\hline Bilateral vocal cord palsy & 01 \\
\hline Suglottic web & 01 \\
\hline Total & 14 \\
\hline
\end{tabular}

Acquired causes of stridor

\begin{tabular}{|l|c|}
\hline Cause & No. of patients \\
\hline Foreign bodies & 03 \\
\hline Post intubation injury & 02 \\
\hline Squamous papilloma & 01 \\
\hline Subglottic haemangioma & 01 \\
\hline Diphtheria & 01 \\
\hline
\end{tabular}

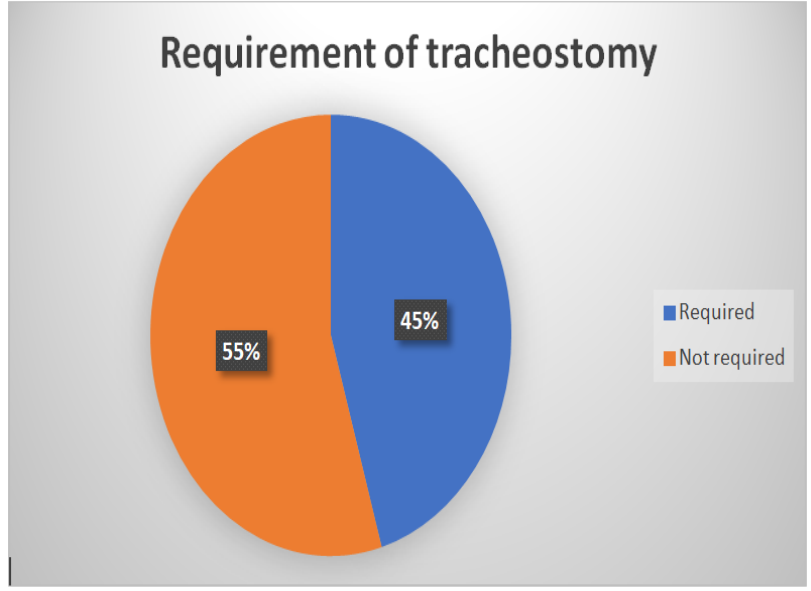

Cases diagnosed by

endoscopyalone

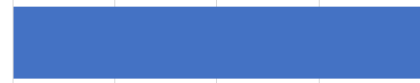

imaging studies+endoscopy

\section{Discusion}

This is a study of evaluation of cause of stridor in neonates, infants and children conducted in Tertiary care Hospital, Visakhapatnam during March 2017 to March 2018.the present study is was undertaken on patients of age group 0-14 yrs. Total of 22 patients were evaluated during this period amongst whom below one month of age were three patients, between two months to twelve months of age were 10,between 2 to 14 years were 9 patients.All the patients in the study were in the age group of below 7 yrs.most of the patients in the study were below one year of age.

Most of the patients were males (13 patients). foreign bodies were common in males (M:F, 2:1). vocal cord palsy was common in females. incidence of laryngomalacia was equal in both the sexes.patients being 5 cases males 5 cases females out of total of 10 cases. 12 out of 22 patients presented with chronic stridor,10 patients presented with acute stridor.they came to hospital 
between hours to 4 months after the onset of stridor.foreign body in trachea and glottis, subglottic haemangioma, subgloticc squamous papilloma, diptheritic laryngitis, subglottic web, bilateral abductor palsy cases presented with acute stridor.Immunization history was normal in all patients except in one case in which we encountered diphtheria. birth history was abnormal in 5 cases.

14 patients presented with congenital causes of stridor

\begin{tabular}{|l|c|}
\hline Cause of stridor & No. of patients \\
\hline Laryngomalacia & 10 \\
\hline Unilateral vocal cord palsy & 02 \\
\hline Bilateral vocal cord palsy & 01 \\
\hline Subglottic web & 01 \\
\hline Total & 14 \\
\hline
\end{tabular}

8 patients presented with acquired causes of stridor

\begin{tabular}{|l|c|}
\hline Cause of stridor & No. of patients \\
\hline Forign bodies of trachea & 02 \\
\hline Foreign body glottis & 01 \\
\hline Post intubation injury & 02 \\
\hline Squamous papilloma & 01 \\
\hline Diphtheria & 01 \\
\hline Subglottic haemangioma & 01 \\
\hline Total & 08 \\
\hline
\end{tabular}

In some cases, associated congenital anomalies are seen, meningocele was associated with bilateral abductor palsy.pectus excavatum was associated with two cases of laryngomalacia. ${ }^{5}$ Holinger et al in his study found that $45.2 \%$ of children with stridor had another associated abnormality involving the respiratory tract, prompting the statement that in evaluating stridor, one does not conclude with laryngoscopy but proceeds with an endoscopic examination of the entire tracheobronchial tree.

In this study most commonly the leision was identified in the larynx,amongst which supra glottis leision mainly laryngomalacia was the commonest observation The aetiology of stridor in neonates is usually congenital. In a study examining stridor in 219 patients, Holinger confirmed this, also noting that more than half of those children aged under 2.5 years had laryngeal abnormalities ${ }^{4}$. glottic leision observed were vocal cord palsy, post intubation edema of both vocal cords, foreign body (whistle) between two vocal cords. subglottic leisions observed were web, hae, angioma and squamous papilloma. Both glottis and sub glottis involved in a case of post intubation injury. 2 cases are with foreign bodies in trachea. All the neonates in this study with stridor were evaluated by radiological tests and by direct examination by endoscopy.plain $\mathrm{x}$ rays of neck and chest did not reveal any abnormality.but CT scan is helful in some cases like subglottic web and subglottic squamous papilloma.

Direct examination of larynx and trachea by rigid as well as flexible endoscopy was valuable for evaluation of cause of stridor and useful to know the extent of leision.in all the cases endoscopy revealed the cause and site of leision.

Tracheostomy was required in 10 cases which include, foreign body in trachea and glottis, subglottic squamous papilloma, post intubation injury and subglottic web, bilateral abductor palsy, subglottic haemangioma, diptheritic laryngitis. Two children died in our study,one was with diptheretic laryngitis and other with bilateral abductor palsy with meningocele. these children presented very late in the course of the disease.

\section{Conclusion}

In this study of evaluation of stridor in neonates, infants and children stridor is common below one year age mainly in males.Congenital leisions are more common than acquired cause of stridor. laryngomalacia is the commonest cause followed by foreign bodies in the airway and vocal cord palsy. Endoscopy is the gold standard for evaluation of stridor in neonates,infants and children. CT is helpful in some cases like subglottic web, subglottic haemangioma, subglottic papilloma. Proper immunization, early treatment of infections, educating parents can prevent some deaths due to stridor. Best treatment outcomes results from a multidisciplinary approach with involvement of otolaryngologists, pediatricians and anaesthesiologists. Assessment should start from careful historytaking and clinical 
examination. Persistent symptoms deserve referral to otorhinolaryngologists for flexible and rigid endoscopy.

\section{References}

1. Scott-Brown's otorhinolaryngology Head and Neck Surgery,Eigth edition.

2. R. Dinwiddie, "Congenital upper airway obstruction," Paediatric Respiratory Reviews, vol. 5, no. 1, pp. 17-24, 2004.

3. S. J. Daniel, "The upper airway: congenital malformations," Paediatric Respiratory Reviews, vol. 7, no. 1, pp. S260-S263, 2006.

4. L. D. Holinger, "Etiology of stridor in the neonate, infant and child," Annals of Otology, Rhinology and Laryngology, vol. 89, no. 5, pp. 397-400, 1980.

5. International Journal of Pediatrics Volume 2012, Article ID 859104, 5 pages http://dx.doi.org/10.1155/2012/859104 article on neonatal stridor.

6. PL Dhingra, Ear, Nose and Throat and Head and Neck Surgery,Seventh edition.

7. Bloom DC, Christenson TE, Manning SC, et al. Int J Pediatr Otorhinolaryngol 2005; 69(5): 657-62.

8. Berg E, Naseri I, Sobol SE. The role of airway fluoroscopy in the evaluation of children with stridor. Arch Otolaryngol Head Neck Surg 2008; 134: 415-18.

9. Kulendra K, Mullineux J, McDermott AL, Williams H. Are contrast swallows a relevant investigation for paediatric stridor? Eur Arch Otorhinolaryngol 2013; 270: 969-73.

10. Mok Q, Negus S, McLaren CA, et al. Computed tomography versus bronchography in the diagnosis and management of tracheobronchomalacia in ventilator dependent infants. Arch Dis Child Fetal Neonatal Ed 2005; 90(4): F290-3.
11. Heyer CM, Nuesslein TG, Jung D, et al. Tracheobronchial anomalies and stenoses : detection with low-dose multidetector CT with virtual tracheobronchoscopy: Comparison with flexible tracheobronchoscopy. Radiology 2007; 242: 542-9. 